\title{
Lifting movements in the size-weight illusion
}

\author{
CHRISTOPHER M. DAVIS and WILLIAM ROBERTS \\ Simon Fraser University, Burnaby, British Columbia, Canada
}

\begin{abstract}
The occurrence of the size-weight illusion is related to the manner in which-objects are lifted. When the SWI occurs, the larger of two objects of equal objective weight is usually lifted with greater acceleration, deceleration, and maximum velocity than the smaller one, but to approximately the same height. These differences are not present when the cans feel equally heavy. The relationship of lifting movements to judgments is consistent with the known behavior of proprioceptors which provide sensory input about muscular and movement events.
\end{abstract}

The size-weight illusion (SWI) is exemplified by the persistent and paradoxical tendency for the smaller of two objects of equal weight to feel heavier than the larger. It is a robust illusion; small objects often feel twice as heavy as large ones of equal weight (Cross \& Rotkin, 1975), and it persists in spite of the observer's knowledge of their actual weight. An early but still tenable explanation of the illusion held that the observer expects the larger object to be heavier and therefore lifts it with greater "motor impulse," causing the object to come up quickly and easily compared to the smaller object, to which a "weak motor impulse" is applied (Martin \& Muller, 1899). It was theorized that the relative ease of lifting of the second object provides the information upon which the judgment is made, so that objects which are easily lifted are felt to be lighter. This is an attractive possibility, both because it specifies the means by which expectation is translated to experience and because it makes the SWI a special case of the mechanism by which all comparative weight judgments are made. The general theory states that when the comparison is between the objects of different objective weight, the second is lifted with about the same force as was required to lift the first. If it comes up easily, it is judged to be lighter; if it is a difficult lift, it is judged to be heavier (Muller \& Schumann, 1889). Specifically, in the SWI, the interlift set, or "einstellung," would reflect the force necessary for the first lift with an increment if the second object was larger than the first object or a decrement if it was smaller.

Present knowledge of receptor mechanisms and motor control in the lifting musculature reveals the physiological basis for the sense of "ease of lifting." The observer's knowledge of the weight of objects is largely dependent on proprioceptors within the muscle, which by their nature respond both to weights lifted and to the muscular force of the lift.

This research was supported by NRC of Canada Grant A8611 to the first author. Requests for reprints should be sent to C. M. Davis, Department of Psychology, Simon Fraser University, Burnaby, British Columbia, Canada.
The muscle spindles, located in the bellies of most striate muscles, respond to stretch with increased rates of firing and to increased muscular tension with decreased firing (Granit, 1970), and these changes could serve as "heaviness" input, since afferents have been shown to project to sensorymotor cortex (Oscarsson, 1965).

The sensitivity to stretch of the muscle spindles themselves is under central neural control through small intrafusal muscle fibers, activation of which increases spindle sensitivity (Granit, 1970). In normal voluntary action, both spindle receptors and contractile muscle fibers are prepared for movement: "Muscle spindles are mobilized in willed contractions and by the aid of the stretch reflex of Liddell and Sherrington (1924) automatically influence the motoneurons of the acting muscles. Co-activation of the extra- and intrafusal motor fibers suggests that the spindle component in excitation is included in the expectations related to the accomplishment of motor acts commanded by the will" (Granit, 1972). With the spindle receptors acting both to "set" the lifting musculature and as the sensor of stretch, it is predicted that differences in muscular set would result in both misapplication of force and in an illusory difference in perceived weight. Granit (1972) hypothesizes the following course of events in the lift of the larger object when the SWI occurs: "The stronger command would have caused a greater spindle discharge acting to facilitate the motoneurons. As a consequence the biceps would have produced a stronger and faster contraction while the tendon organs, if playing a role for sensory experience, would have recorded tension objectively. During the actual contraction the spindle activity would have diminished at an unexpectedly fast rate owing to rapidity of the shortening of the muscle."

These events would of necessity result in different lifting movements, at least in the early stages. The larger of two objects would be lifted more forcefully, and that would result in greater early acceleration. Loomis (1907) tied strings to the bottoms of large and small cans and reported that greater energy was 
expended in the lift of the large can. But direct measurement of lifting movements has not been reported.

\section{METHOD}

\section{Subjects}

Six right-handed adults were paid for serving.

\section{Apparatus}

The stimulus objects were 12 modified paint cans, 6 1/2-pt $\left(236 \mathrm{~cm}^{3}\right)$ and $61-\mathrm{qt}\left(946 \mathrm{~cm}^{3}\right)$. Rigid wire handles were of equal width but different heights so that the handles of both large and small cans were $3.2 \mathrm{~cm}$ above the arm of the subject's chair. All cans weighed $500 \mathrm{~g}$. Both cans of a pair were placed on a rotatable circular table so that cans could be moved into lifting position. Blocks of polystyrene, grooved to fit the handles of the can on one side and shaped to the curvature of the finger on the other, were taped to the first and third finger proximal to the terminal phalanx, so that the cans were lifted through the same lever system and the role of skin receptors was minimized.

An instruction panel beyond the lifting platform in the subject's line of vision contained the words "prepare to lift," and "lift" beside jeweled 12-V signal lights which were controlled by appropriate relay timing circuitry.

The entire course of each lift was filmed at 24 frames/sec by a Bolex $16-\mathrm{mm}$ movie camera, Model H-16, Kern-Paillard $16-\mathrm{mm}$ lens, f 2.8 , tripod mounted, so that the lens was $1.2 \mathrm{~m}$ away at the same height as the object lifted. The processed films were projected one frame at a time, and the displacement of the can from the platform was measured for each frame (equivalence of recording and measurement displacement scales was established by filming a vertically mounted standard millimeter scale in the plane of the cans at the start of each session).

\section{Procedure}

The subject was seated and his right forearm taped, palm up, to the padded arm of the chair so that wrist and finger flexion were possible. After the lifting guides were affixed, the subject practiced lifting a dummy can until the manner of approaching and lifting was consistent and reasonably comfortable. The subjects was instructed that he would be lifting pairs of cans following the "prepare" and "lift" instructions and to report, following the second lift of a pair, whether the second can felt heavier than, equal to, or lighter than the first. Ten pairs of cans, a large and a small one to each, were then presented with a preparation period of $.5 \mathrm{sec}$ before each lift, an interlift interval of 8 to $12 \mathrm{sec}$, and an interpair interval of $30 \mathrm{sec}$. The large can was first on five trials selected randomly. Cans were drawn from a box visible to the subject in a pseudoorganized order. Both cans of a pair were placed on the lifting platform before the trial began.

\section{RESULTS}

The SWI was present on $63 \%$ of the trials and absent on the remainder, except for one trial for a single subject where the large can felt heavier, a reversal of the SWI. The average course of the lifts with respect to time is shown in Figure 1, which reveals almost identical average peak heights when the illusion occurred and a large mean difference when it did not. This difference was significant, $F(1,19)=4.69, \mathrm{p}<.05$, but can hardly serve as a contributor to the illusion since it occurs only when the illusion does not. However, peak height is the summation of movements which precede it, and those movements would best reflect the muscular forces applied initially. Peak velocity, shown in Figure 2 , usually occurred early in the lift and was significantly greater for the large can when the illusion occured $F(1,5)=9.33, p<.05$, but not when the SWI failed. The greater average peak velocity would be expected to result from greater acceleration early in the lift, since the cans were starting from the same point at the same time with respect to the beginning of the lift. Indeed, when the SWI occurred, there was significantly greater peak acceleration of the large can, $F(1,5)=19.40, p<.01$. A difference in early acceleration was apparent when the illusion failed, but was not significant. But when the illusion occurred, the mean course of the lift included a quicker deceleration of the large can, $F(1,32)=4.90, p<.05$, which explains the small difference in peak height when the illusion occurred. Both initial acceleration and rate of subsequent deceleration are important to the occurrence of the illusion. When there was either greater peak acceleration or deceleration of the large can, the illusion was present on 20 occasions and absent on 4 . When the large can was lifted with neither greater acceleration nor deceleration, the illusion was present on 13 lift pairs and absent on 15 .

Usually, then, when the SWI occurred, the larger can was lifted with greater peak acceleration than the smaller can and reached a greater peak velocity, but was quickly brought to a stop, so that the cans were at nearly the same mean height at their peak and were then lowered at about the same rate.

\section{DISCUSSION}

These results can be taken both as support for a motor theory of the SWI and as a specification of the role of movement. The differences in early acceleration found here would necessarily result from different forces being applied to identical masses, and are consistent with Granit's (1972) description of muscular and proprioceptive events preceding the movement. Indeed, the very quick (less than $0.24 \mathrm{sec}$ ) compensatory deceleration of the large can when the illusion occurred is suggestive of a reflex response to a sudden reduction in spindle output. The similarity of movement of the two cans as early as $.6 \mathrm{sec}$ into the lift suggests reflex recovery to a "planned movement pattern."

However, the illusion did often occur without measured differences in acceleration or deceleration and sometimes did not occur when there were movement differences. But our measurements were imperfect, since changes in displacement could be examined only 24 times each second, a relatively long time in physiological terms. More importantly, the lifting movements, while properly representing 

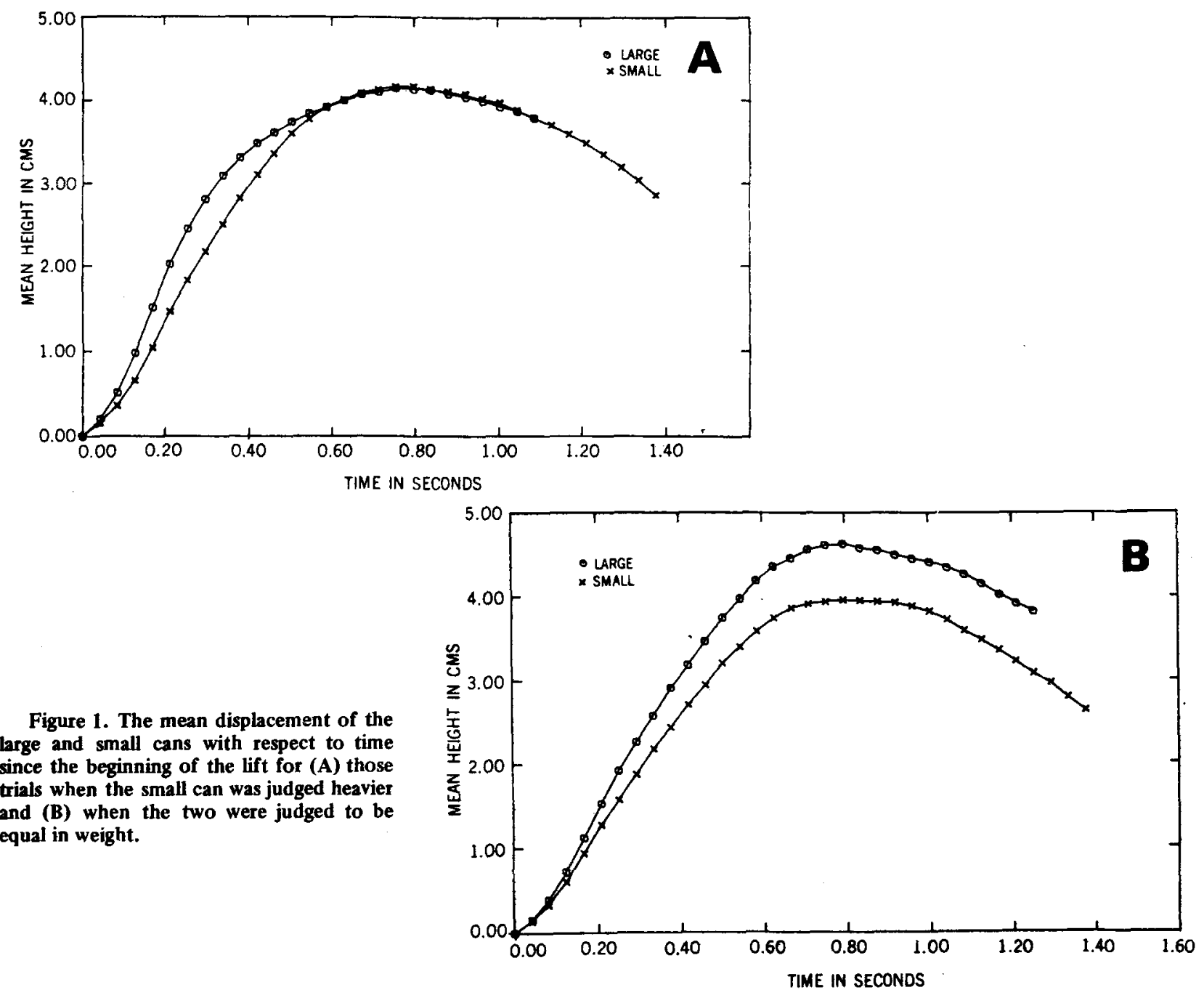

Figure 1. The mean displacement of the large and small cans with respect to time since the beginning of the lift for (A) those trials when the small can was judged heavier and (B) when the two were judged to be equal in weight.

SECONDS 
the sum of all muscular forces acting, need not perfectly reflect the physiological events upon which judgments are based, unless, of course, the movements themselves are crucial. Since many muscles are involved in wrist flexion and their combined action changes during flexion, the relation of movement events to perceptual ones is not simple.

The relationships between movement and perceived heaviness reported here, are correlational; the illusion could be a result of some other factor, a central "set to perceive," for instance, of the sort proposed by Koseleff (1957), which causes both movements and illusion. However, if that were the case, then the differences in proprioceptive input which accompany the movement differences found here would need to be dealt with. It would seem that both parsimony and the appeal of physical explanation argue that a misapplication of lifting forces through the gamma efferent system is at the base of movement and illusion. Furthermore, the SWI was reduced in frequency, and often reversed, when a large can was lifted from the fingertips and a small one from the base of the fingers (Davis, 1973). There the misapplied force was countered by altering the mechanical advantage of the lifting system so as to reduce the difference between the force applied and the resulting movement.

The explanation of the illusion offered here can be extended to other illusory experiences of movement and perception. The apparent density illusion (Harshfield \& DeHardt, 1970), in which a cube of balsa wood felt heavier than a hollow cube of identical size and weight but machined from brass, would be expected to result from the same mismatch of pro- prioceptive set and resulting movement. Granit lists at least nine more, all based on unexpected spindle activity. "These suggest that co-activated spindles, which are the only end organs reflecting both demand and execution, play a most essential role in our judgments about muscular exertion, difficult though it may be to formulate the proprioceptive experience in the way we can describe things seen or heard"' (1972, p. 656).

\section{REFERENCES}

Cross, D. V., \& Rotkin, L. The relation between size and apparent heaviness. Perception \& Psychophysics, 1975, 18. $79-87$.

DAvis, C. M. Mechanical advantage in the size-weight illusion. Perception \& Psychophysics, 1973, 13, No. 2, 238-240.

Granit, R. The basis of motor control. New York: Academic Press, 1970.

GRANit, R. Constant errors in the execution and appreciation of movement. Brain, 1972, 95, 649-660.

Harshfield, S. P., \& DEHARdt, D. C. Weight judgement as a function of apparent density of objects. Psychonomic Science, 1970, 20, 365-366.

KoselefF, P. Studies in the perception of heaviness. I. Acta Psychologica, 1957, 13, 242-252.

Loomis, H. N. Reactions to equal weights of unequal size. Psychological Monographs, 1907, 8, 334-338.

Martin, L., \& Muller, G. E. Zur Analyse der Unterschiedsempfindlichkeit. Leipzig: 1899.

Muller, G. E., \& Schumann. F. Ueber die psychologischen Grundlagen der Vergleichung gehobener Gewichte. Archives für die Gesamte Physiologie, 1889, 45, 37-112.

Oscarsson, $O$. Functional organization of the spino- and cuneo-cerebellar tracts. Physiological Review, 1965, 45, 495-522.

(Received for publication January 12, 1976; revision received April 28, 1976.) 